\title{
Recurrent Breast Cancer
}

\author{
Treatment Strategies for Maintaining and Prolonging Good Quality of Life
}

\author{
Bernd Gerber, Mathias Freund, Toralf Reimer
}

\section{SUMMARY}

Background: Recurrent breast cancer remains a challenge for interdisciplinary treatment even though new therapeutic options are available.

Methods: The PubMed database was selectively searched for articles that appeared from 1999 to 2009 and contained the key words „breast cancer,“ „recurrence,“ „metastatic,“ „advanced,“ and „treatment". Further sources consulted for this review included the German S3 guideline, the treatment recommendations of the German AGO-Mamma group, the NCCN guidelines, and the Cochrane database.

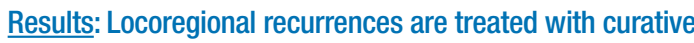
intent. Metastatic breast cancer must be treated on an individualized basis: The treatment should be continued as long as its benefits for the individual patient outweigh its adverse side effects. Endocrine treatment is indicated for all patients whose tumors are hormone-receptor positive or of unknown receptor status and who have enough time for a response to be seen. Chemotherapy should be given if the tumor is hormone-receptor negative, if a rapid response is urgently needed, or if endocrine treatment has failed to produce a response. Combination chemotherapy improves response rates and prolongs progression-free survival, yet it does not prolong overall survival in comparison to monochemotherapy. In HER2-positive patients, first-line treatment with trastuzumab and monochemotherapy prolongs overall survival. Other treatment options include angiogenesis inhibitors, various tyrosine kinases inhibitors, radiotherapy, bisphosphonates, surgical or other ablative treatment of metastases, or a combination of these approaches, applied either simultaneously or consecutively.

Conclusions: While locoregional recurrences of breast cancer should be treated with curative intent, breast cancer with distant metastases is currently not curable. It is treated with the intention of restoring and maintaining good quality of life and relieving symptoms due to the metastases, rather than prolonging survival.

\section{Cite this as: Dtsch Arztebl Int 2010; 107(6): 85-91} DOL: 10.3238/arztebl.2010.0085

Universitätsfrauenklinik am Klinikum Südstadt der Hansestadt Rostock: Prof. Dr. med. Gerber, PD Dr. med. Reimer

Klinik und Poliklinik für Innere Medizin, Abteilung Hämatologie und Onkologie der Universität Rostock: Prof. Dr. med. Freund

\begin{abstract}
$\triangle$ t present around $40 \%$ of all patients with breast cancer suffer a recurrence; most of them die from it (1, e1-e3). Breast cancer thus remains the most common cause of cancer-related death in women. The risk of recurrence is highest in the first 2-3 years and then decreases continuously, although it never reaches zero (e4). Ten percent to $20 \%$ of all recurrences are isolated locoregional recurrences, while $60 \%$ to $70 \%$ are distant metastases in one "anatomical structure," or else in multiple locations (2, e4). The incidence and location of recurrences depend on the initial tumor stage, previous therapy, tumor biology, and the sensitivity of the diagnosis (Table) $(1,3,4$, e5, e6) (Cheang et al.: Breast cancer molecular subtypes and locoregional recurrence. J Clin Oncol [Proceedings of ASCO] 26, [May 20 Suppl; Abstr 510] 2008). This article will give a systematic overview of treatment for recurrent breast cancer.
\end{abstract}

\section{Materials and methods}

A selective literature search was carried out in the PubMed database using the search terms "breast cancer" and "recurrence," "metastatic," “advanced," and "treatment" for the period from 1999 to January 2009. Contributions to international congresses on breast cancer in 2008 (ASCO, American Society of Clinical Oncology; SABCS, San Antonio Breast Cancer Symposium; ECCO, European Cancer Organisation; EBCC, European Breast Cancer Conference) were also included. The current German S3 Guidelines (5), the treatment recommendations of AGO-Mamma (the Breast Group of the German Gynecological Oncology Working Group, the Arbeitsgemeinschaft Gynäkologische Onkologie, Organgruppe Mamma) (6) and the American NCCN (National Comprehensive Cancer Network) Guidelines (7) together with the Cochrane Database (8) were also included. The statements were evaluated-if this had not already been done in the guidelines themselves-according to the Oxford criteria (evidence level, EL) (e7) and the AGO recommendation grades (Box 1).

\section{General recommendations}

Since hormone-receptor expression and HER2 expression can change in the course of metastasization, determination of receptor status should always be carried out when recurrence occurs, if reasonably possible (EL 1/A AGO-GR++) (e8). In order to detect any further metastases, a re-staging procedure is 
TABLE

Location and incidence of metastases found clinically and at autopsy in patients with metastatic beast cancer $(2,4)$

\begin{tabular}{l|l|l}
\hline Location & $\begin{array}{l}\text { Clinical } \\
\text { findings (\%) }\end{array}$ & $\begin{array}{l}\text { Autopsy } \\
\text { findings (\%) }\end{array}$ \\
\hline Brain & $5-10 \%$ & $30-50 \%$ \\
\hline Lung/pleura & $15-20 \%$ & $50-75 \%$ \\
\hline Local/regional & $20-40 \%$ & $30-50 \%$ \\
\hline Heart & $<5 \%$ & $25-40 \%$ \\
\hline Liver & $5-15 \%$ & $50-75 \%$ \\
\hline Bone & $20-60 \%$ & $60-90 \%$ \\
\hline Intra-abdominal & $<5 \%$ & $30-40 \%$ \\
\hline Endocrine/ovaries & $<5 \%$ & $20-40 \%$
\end{tabular}

BOX 1

Recommendation grades of the Breast Group of the Gynecological Oncology Working Group (AGO-Mamma) (6)

++ This therapy or intervention is highly beneficial for patients and should be performed.

$+\quad$ This therapy or intervention is of limited benefit for patients and may be performed.

+/- This therapy or intervention is not of benefit for patients but may be performed in individual cases.

- $\quad$ This therapy or intervention can be disadvantageous for patients and ought not to be performed.

-- $\quad$ This therapy or intervention has clear disadvantages for patients and should not be performed under any circumstances. recommended (chest radiography, bone scintigraphy and liver ultrasonography) (EL 5/D AGO-GR++), although this has not be shown to carry any survival advantage for the patient.

\section{Locoregional recurrence}

Local disease recurrence (Box 2) is generally treated curatively (9). In some cases it can be difficult to distinguish between a locoregional recurrence and an ipsilateral second tumor. Features suggesting a second tumor-which like primary breast cancer should be treated curatively-are:

- A long interval of time since the first tumor

- A different location in the breast

- Different tumor biology (hormone-receptor status, HER2-receptor status, tumor grade).

Five-year overall survival after an isolated chest wall recurrence is $68 \%$; after intra-breast recurrence it is $81 \%$ (e4). Operable breast, chest wall, and axillary recurrences should be excised with tumor-free margins (EL 2b/A; AGO-GR++). For intra-breast recurrence, mastectomy is regarded as the standard treatment, although in some cases repeat breast-preserving surgery and interstitial radiotherapy may be undertaken (EL 3/C; AGO-GR+/-). The rate of repeat intra-breast recurrence is higher after such treatment (e9), but the significance of this for overall survival is unclear (6). Patients who have not yet received radiotherapy should be offered it (EL 2b/B; AGO-GR+).

Antihormonal therapy after $\mathrm{R} 0$ resection of a locoregional recurrence with $\mathrm{M} 0$ status has prolonged the interval until a repeat recurrence, but without improving overall survival (EL 5/D; AGO-GR++) (10). No valid study results are available for chemotherapy or trastuzumab therapy after R0 resection of a local recurrence, so that these cannot be definitely recommended at present (EL 3b/C; AGO-GR+/-) (e10, e11). The exception to this is in HER2-positive patients who have not yet received anti-HER2-treatment (trastuzumab or lapatinib); in this group anti-HER2-treatment (trastuzumab or lapatinib) can be recommended (EL 5/D; AGO$\mathrm{GR}+$ ).

In patients with R1-resection in whom further resection is not possible, and in other cases of locoregional recurrences (lymph nodes, skin), chemotherapy, monoclonal antibody therapy, radiotherapy (EL 2/B; AGO$\mathrm{GR++}$ ) and combinations of these (e.g., simultaneous radiochemotherapy, $\mathrm{EL} 3 \mathrm{~b} / \mathrm{C}$; $\mathrm{AGO}-\mathrm{GR}+$ ) may be considered (e12, e13). In patients with M1 status and/or inoperable local recurrence, decisions about palliative surgery must be made on an individual basis (e.g., where there is ulceration, unpleasant odor, or pain) (EL 5/D; AGO-GR+/-) (6).

\section{Metastatic breast cancer Treatment goals}

Because metastastic breast cancer is not curable, the primary goals of therapy are restoration of quality of life, reduction of tumor-related symptoms, and maintenance of the patient's social environment; prolonging life is secondary to these (EL 1a/A; AGO-GR++). The possibility of increasing the overall survival of patients with metastastic breast cancer has been the subject of debate in recent years (e14). However, prospective and retrospective studies show that the treatment options available at present do improve overall survival (EL 2a) (e15). The median survival of patients with metastastic breast cancer is currently given as 20 to 28 months; it depends heavily on the nature of the metastases and the tumor biology (11, 12, e16-e18).

\section{Treatment monitoring}

To monitor the efficacy of antitumor treatment, a "marker lesion" is chosen. This should be monitored with the simplest method of examination-palpation, ultrasonography, or tumor marker evolution (if raised; CA15-3, CEA, or CA-27-29; HER-2 shed antigen, ECD. Tumor response is "objectified" according to the RECIST criteria (response evaluation criteria in solid 
tumors), whereby the longest diameter of the target lesion that can be best visualized is monitored over time-depending on the clinical situation, every 2 to 3 months (e19). Side effects must be recognized before they become clinically manifest (e.g., using echocardiography during trastuzumab therapy) (e20).

\section{Available treatments}

Currently licensed treatment options have been greatly expanded in recent years (Box 3). In the choice of therapy, previous treatments, comorbidities, side effects, and also the expectations and wishes of the patient must all be taken into account (EL 1c/A; AGO-GR++). More significant than the patient's actual age is the function of her individual organs (bone marrow, kidneys, liver, heart). Dosages should follow those of the trials on which the licensing was based (EL 1c/A; AGO-GR++). All patients should be offered the chance to participate in clinical studies (AGO-GR++).

\section{Primary metastatic breast cancer}

The prognosis of primary metastatic breast cancer depends on the location of the metastases, but is generally regarded as poorer than that of secondary metastatic tumors (EL 4/C). In primary metastatic breast cancer, resection with tumor-free margins (tumorectomy, mastectomy) improves 5-year overall survival by $40 \%$ to $50 \%$ (13, e21, e22). The role of axillary surgery is unclear.

\section{Endocrine (antihormonal) treatment}

Today, endocrine treatment is the first line of therapy for all patients with metastatic breast cancer and positive or unknown hormone receptor status whose disease is not at a life-threatening stage (EL 1a/A; AGOGR++). A delay of 10 to 12 weeks must be allowed for before the endocrine treatment will start to take effect. Extensive visceral metastases, CNS metastases and/or an urgent need for remission (pain) are reasons not to undertake endocrine treatment. Concurrent chemoendocrine therapy (EL 1b/A; AGO-GR- -) must be avoided, as this is no more effective than the separate therapies but has more side effects (14). Endocrine maintenance therapy following a response to chemotherapy improves not only disease-free survival but also overall survival (EL 3/C; AGO-GR++) (e23).

\section{HER2-positive metastatic breast cancer}

HER2 status is not in itself a decision criterion for or against endocrine treatment. All endocrine therapies are less effective when there is overexpression of HER2 than when there is not (15). Compared to anastrozole treatment alone the combination of anastrozole with trastuzumab significantly improved the remission rate (20\% versus $7 \%$ ) and the progression-free interval (5 versus 2 months) (EL 2b/B; AGO-GR+/-) (16).

Compared with an aromatase inhibitor alone, in patients not previously treated with trastuzumab, letrozole combined with the dual tyrosine kinase (HER2, EGFR) inactivator lapatinib showed a significantly

\section{BOX 2}

\section{Definition of locoregional recurrence (9)}

\section{Recurrence of disease:}

- In the breast (after breast-preserving therapy)

- In the chest wall (after mastectomy)

- In the ipsilateral/parasternal/infra- or supraclavicular lymph nodes

- In the skin of the chest wall (not breast)

- In the reconstructed breast

- As a second carcinoma (e.g., angiosarcoma)

\section{BOX 3}

\section{Current treatment options in metastatic breast cancer}

1. Endocrine therapy (tamoxifen, aromatase inhibitors, fulvestrant, gestagens, GnRH analogs)

2. Chemotherapy (anthracyclines, taxanes, capecetabine, vinorelbine, etc.)

3. Monoclonal antibody therapy (trastuzumab, bevacizumab)

4. Tyrosine kinase inhibitors (lapatinib)

5. Bisphosphonates

6. Irradiation

7. Operative/ablative procedures

8. Combinations/sequences

9. Other (VATS pleurodesis) (VATS: video-assisted thoracic surgery

higher remission rate (15\% versus $28 \%$ ) and a longer progression-free interval (3 versus 8 months; EL 2b/B; AGO-GR+/-) (17).

Nevertheless, the combination of chemotherapy with trastuzumab remains the standard for all HER2positive patients. Combinations of hormone therapy and trastuzumab should only be considered for selected patients (e.g., with comorbidities or in whom chemotherapy is contraindicated).

\section{HER2-negative metastatic breast cancer}

For premenopausal women, the standard treatment is suppression of ovarian function combined with a second endocrine treatment step (aromatase inhibitor, fulvestrant) by analogy to postmenopausal patients (e24-e26). Endocrine treatment in postmenopausal 


\section{Endocrine treatment of metastatic breast cancer, depending on previous treatments}

\section{During or after adjuvant tamoxifen treatment}

All third-generation aromatase inhibitors were more effective as first-line treatment in respect of remission rates and progression-free survival than any other endocrine treatment (EL 1a/A; AGO-GR++) (e62). Whether fulvestrant or, depending on the aromatase inhibitor used in first-line treatment, a steroidal (exemestane) or non-steroidal aromatase inhibitor (anastrozole, letrozole) should be used as second-line treatment must be decided individually in each case. No cross-resistances exist so giving steroidal after non-steroidal aromatase inhibitors, with a clinical success rate of $25 \%$ to $47 \%$, has comparable effectivity to giving the same substances in the reverse order (e63-e65).

Exemestane was also effective as third-line treatment, with a 40\% rate of clinical success (e66). Reasons for early use of fulvestrant (selective estrogen-receptor downregulator, SERD) are its very good side effect profile (no musculoskeletal symptoms) and its higher clinical success rate (first-line treatment 57\%, second-line treatment 46\%) (EL 2b/B; AGO-GR+) (e66, e67).

\section{During upfront treatment with aromatase inhibitors (adjuvant from the start)}

After treatment with non-steroidal aromatase inhibitors, the steroidal aromatase inhibitors and fulvestrant (EL 2b/B; AGO-GR+) were of equal value, with a response rate of $7 \%$, a clinical success rate of $32 \%$ of treated cases, and a progression-free interval of 9 months (e69). There are no data relating to the use of tamoxifen in this situation.

\section{During treatment with aromatase inhibitors after switch therapy}

( 2 to 3 years tamoxifen followed by aromatase inhibitor)

If there has been a long interval since earlier tamoxifen treatment, tamoxifen would be an alternative to fulvestrant and the non-cross-resistant aromatase inhibitor (EL 4/D; AGO-GR+).

\section{During extended therapy with an aromatase inhibitor ( 5 years tamoxifen followed by aromatase inhibitor)} In situations where data are missing, fulvestrant or the non-cross-resistant aromatase inhibitor would be a treatment option (EL 4/D; AGO-GR+).

patients with metastatic breast cancer depends on which treatment was previously given, and for how long. At present, there are four clinical situations (Box 4). After treatment with tamoxifen, aromatase inhibitors, and fulvestrant, gestagens (medroxyprogesterone acetate, megestrol acetate), with their anabolic, analgesic, and euphorizing side effects, show positive effects in patients in the final stage of the disease. Otherwise, endocrine treatment starts again from the beginning (EL 5/D; AGO-GR+).

\section{Chemotherapy and targeted therapy}

There are no real predictors of responsiveness to chemotherapy. If chemotherapy is indicated (Box 5), it should be continued so long as the therapeutic index is favorable, i.e., so long as the benefit is greater than the side effects (EL 2b/B; AGO-GR+). Disease progression and untreatable side effects are signs that warrant treatment cessation (EL 1c/A; AGO-GR++).

\section{Mono- versus polychemotherapy}

A meta-analysis from 1996 that covered 996 patients and two randomized studies (docetaxel, D, versus docetaxel+capecitabine, DC; paclitaxel, $\mathrm{P}$, versus paclitaxel+gemcitabine, PG) compared monochemotherapies to combination chemotherapies (e27-e29). This showed the combination to be superior to the monotherapy in respect of response (D versus DC: $30 \%$ versus 42\%; P versus PG: 26\% versus 41\%), progression-free interval (4 versus 6 months in both cases), and overall survival (D versus DC: 12 versus 15 months; P versus PG: 16 versus 19 months). However, the combination treatments also led to significantly more side effects. These studies did not address the question of sequential administration of the individual substances. In a multicenter three-arm study, 739 patients were randomized to receive doxorubicin, paclitaxel, or both (A, P, AP) (18). When progression occurred, the patients in the monotherapy arms were treated with the substance they had not received so far. The combination gave rise to higher remission rates (A $34 \%$, P 36\%, AP 47\%) and a longer progression-free interval (A 6 months, P 6 months, AP 8 months), though toxicity was also higher. There were no significant differences in total survival in the three arms (A 19 months, P 22 months, AP 22 months). At present there are few indications that justify combination chemotherapy, especially since there is no guarantee of a survival advantage in comparison to sequential monotherapy with the same substances (e30).

\section{HER2-negative metastatic breast cancer}

The first-line treatment with the highest recommendation grade for previously untreated metastatic breast cancer is anthracyclines and taxanes (EL 1b/A; AGO$\mathrm{GR}++$ ) (6). Docetaxel appears to be better when given 
at 3-weekly intervals and paclitaxel at weekly intervals (6, 19, e31, e32). Many patients have already received anthracyclines and taxanes as adjuvant treatment. If there has been a long period (more than 2 to 3 years) between the adjuvant treatment and metastatic recurrence, it is certainly acceptable to give a taxane or anthracycline. To reduce the risk of cumulative cardiotoxicity, in this situation the liposomal encapsulation of doxorubicin or epidoxorubicin can be used (EL 1b/A; AGO-GR++). When the patient has been previously treated with anthracyclines, taxanes are indicated (EL 1a/A; AGO-GR++); after previous treatment with anthracyclines and taxanes, capecitabine (EL 2b/B; AGOGR++), vinorelbine, nab-paclitaxel (nanoparticlealbumin-bound paclitaxel, licensed in the USA), and PEG-liposomal doxorubicin are indicated (EL 2b/B; AGO-GR+). Vinorelbine is indicated above all when there are contraindications to treatment with taxanes (e.g., impaired liver function). Cytostatics not licensed in Germany are nab-paclitaxel and ixabepilone (e33-e35) (licensed in the USA; EMEA, the European Medicines Agency, did not approve its licensing in 2008). Ixabepilone works via inhibition of the microtubule system, without the occurrence of crossresistance with taxanes (e36-e38). For off-label use of ixabepilone, neuropathy and the therapeutic index must be monitored. Neither of these substances requires a solubilizer, so premedication is unnecessary. If polychemotherapy is indicated, anthracycline-taxane combinations are recommended in patients not previously treated with anthracycline (EL 1b/A; AGOGR++), and combinations of taxanes with capecitabine (EL 2b/B; AGO-GR+) or gemcitabine (EL 2b/B; AGO$\mathrm{GR}++)$ in patients who have previously received anthracyclines (e28, e29).

Vascularization is a necessary part of metastasization. To that extent, inhibition of angiogenesis using the VEGF-specific antibody bevacizumab is a logical therapeutic approach. As first-line treatment in HER2-negative patients, bevacizumab in combination with docetaxel (D, once every 3 weeks) or paclitaxel (P, once a week) led to significantly higher remission rates (D: $63 \%$ versus 44\%, P: $28 \%$ versus $14 \%$ ) and an increase in progression-free survival (D: 1 month, P: 5 months), although overall survival was not affected (EL 1b/B; AGO-GR+) (20) (Miles et al.: Randomized, double-blind, placebo-controlled, phase III study of bevacizumab with docetaxel or docetaxel with placebo as first-line therapy for patients with locally recurrent or metastatic breast cancer [mBC]: AVADO. Proc Am Soc Clin Oncol 26, abstr. LBA1011. 2008).

The efficacy and side effects of bevacizumab depend on its early use and individual VEGF genotypes (e62). Bevacizumab is licensed for use in combination with taxanes for first-line treatment of HER2-negative metastatic breast cancer.

\section{HER2-positive metastatic breast cancer}

Patients who overexpress HER2 benefit from early use of the monoclonal antibody trastuzumab in combination
BOX 5

\section{Indicators for chemotherapy}

- Urgent need for remission (aggressive disease progression, symptoms, vital functions at risk)

- Negative hormone receptor status

- When monoclonal antibody treatment is indicated

- When endocrine treatment is followed by no response or by disease progression

with docetaxel or paclitaxel, with a significant increase in median overall survival (D: 23 versus 31 months, $P$ : 20 versus 25 months; EL 1b/A; AGO-GR++) (21, e40). For this reason, trastuzumab in combination with a cytostatic should be offered as first-line therapy to all patients, with the exception of older patients and those with cardiac morbidity (EL 1b/A; AGO-GR++). Monitoring of heart function is obligatory during trastuzumab therapy. If progression occurs during trastuzumab treatment ( \pm taxane), the present recommendation is to switch to lapatinib+capecitabine (EL 1c/B; AGO-GR+), since the combination of lapatinib+capecitabine is significantly better than chemotherapy alone in terms of tumor response (29\% versus $16 \%$ ) and progression-free survival (37 versus 20 weeks) (e41, e42). Lapatinib ( \pm capecitabine) seems to be effective in the treatment of radiotherapy-resistant brain metastases (EL 2b/B; AGO-GR+/-) (e43). In patients with metastatic breast cancer not previously treated with trastuzumab, the combination of lapatinib+paclitaxel (EL 2b/B; AGO-GR+/-) led to a significant improvement in clinical benefit (69\% versus $40 \%$ ) and progression-free survival (36 versus 25 months) compared to chemotherapy alone, and median overall survival was also increased (105 versus 82 months) (22). Prospective studies on continuing trastuzumab when disease progression occurs during trastuzumab therapy in combination with chemotherapy showed that continuing the antibody treatment and changing the chemotherapy had a significant advantage over chemotherapy alone, leading to a treatment response of $49 \%$ versus $25 \%$ for the combination (e44). Continuing trastuzumab therapy when disease progression occurs and changing the chemotherapy (EL 2b/B; AGO-GR+) represents an alternative to lapatinib+chemotherapy. Clinical trials are now ongoing into another HER2 monoclonal antibody, pertuzumab (e45).

\section{Bisphosphonates}

Bisphosphonates (BPs) are indicated in all patients with bone metastases (EL 1a/A; AGO-GR++). By inhibiting osteoclast activity, BPs lead to bone stabilization, a reduction in serum calcium concentration, and avoidance of skeletal complications (23, e46-e51). BPs are 
given in combination with antitumor therapy and should continue to be given after disease progression (EL 5/D; AGO-GR++). The oral and intravenous routes are equally effective (23, e49-e52). Osteonecrosis of the jaw during intravenous administration (EL 2b) can be avoided if dental cleaning and restoration is carried out first, or by oral administration (EL 4/C; AGO$\mathrm{GR}+$ ). More recent data suggest that BPs have direct antitumoral and bone-protective effects (EL 1b/A; AGO-GR+) (24). Denusomab (not yet licensed), a subcutaneously administered anti-RANKL (receptor activator of nuclear factor kappaB ligand) antibody, showed a positive effect on bone metabolism with fewer side effects than BP (e53).

\section{Radiotherapy}

Irradiation of symptomatic bone metastases leads to an improvement in pain symptoms, in mobility and function, local stabilization, and reduced risk of bone fracture (EL 1a/B; AGO-GR++).

However, in cases where there is spinal compression or risk of fracture, the combination of surgery followed by radiotherapy is superior to radiotherapy alone (EL 3b/C; AGO-GR++) (e54). For this reason, before radiotherapy is started, the question of whether operative stabilization is required should be clarified at a multidisciplinary case conference (e55).

In cases of "small” CNS oligometastases or where there are metastases in an unfavorable (inoperable) location, MRI-guided stereotactic single irradiation ("radiosurgery") or fractionated irradiation should be carried out (EL 2b/B; AGO-GR++) (e56). After surgery on CNS metastases, percutaneous irradiation of the entire cranium improves local control and overall survival (EL 2b/B; AGO-GR++). Where there are multiple CNS metastases, irradiation of the entire cranium accompanied by antiedema therapy (glucocorticoids) (EL 1a/A; AGO-GR++) or simultaneous radiochemotherapy (temozolomide, topotecan) is an option (EL 3b/C; AGO-GR+/-). For the treatment of skin, lymph node, plexus, and spinal canal metastases, irradiation with or without combined chemotherapy (EL 4/D; AGO-GR+/-) is effective (e12).

\section{Operative/ablative treatment}

For patients with "small” oligometastases who are in good general condition and have had a long progression-free interval, operative or ablative procedures (RFA, LITT, cryotherapy) are therapeutic options (EL 3b/C; AGO-GR+/-) that should be carefully weighed in each individual case (e57-e59). It should be pointed out that these results come from case series with highly selected patients. In addition, the effect of growth factors on tumor cells after major invasive procedures is unclear (e60).

\section{Other treatment options}

At present there are no recommendations for regional chemotherapy or the use of hyperthermia (EL 3b/C; AGO-GR-). The treatment of choice for pleural effusion is pleurodesis with talcum and VATS (videoassisted thoracic surgery) (EL 1b/B; AGO-GR++) (25, e61).

\section{Future prospects}

In the present authors' view, metastatic breast cancer will remain a great challenge for physicians and researchers. Although long-term remissions are possible in individual cases today, no treatment will be available in the foreseeable future that can claim to be curative. For this reason the main aim of current treatment for metastatic breast cancer continues to be, as the authors said at the beginning, to restore and maintain quality of life by the alleviation of symptoms caused by metastases.

\section{Conflict of interest statement}

Professor Gerber has received lecture fees and reimbursement of travel expenses from Astra Zeneca, Pfizer, Novartis, Roche, GSK, and Sanofi-Aventis. Dr. Reimer has received lecture fees and reimbursement of travel expenses from Novartis, Astra Zeneca, Pfizer, Sanofi-Aventis, and Roche.

Professor Freund has received lecture fees and reimbursement of travel expenses from Hoffmann-La Roche, Novartis, Janssen-Cilag, Astra Zeneca, Mundipharma, and Bristol-Myers Squibb.

Manuscript received on 12 January 2009, revised version accepted on 17 June 2009.

Translated from the original German by Kersti Wagstaff, MA

\section{REFERENCES}

1. Jemal $A$, Thun MJ, Ries $L A$, et al.: Annual report to the nation on the status of cancer, 1975-2005, featuring trends in lung cancer, tobacco use, and tobacco control. J Natl Cancer Inst 2008; 100: 1672-94.

2. Kamby C, Vejborg I, Kristensen B, Olsen LO, Mouridsen HT: Metastatic pattern in recurrent breast cancer. Special reference to intrathoracic recurrences. Cancer 1988; 62: 2226-33.

3. Rhee J, Han SW, On DY, et al.: The clinicopathologic characteristics and prognostic significance of triple-negativity in node-negative breast cancer. BMC Cancer 2008; 8: 307

4. Kamby C, Ejlertsen B, Andersen J, et al.: The pattern of metastases in human breast cancer. Influence of systemic adjuvant therapy and impact on survival. Acta Oncol 1988; 27: 715-9.

5. DGS: S3-Leitlinie der Deutschen Gesellschaft für Senologie. 2008; http://www.senologie.org

6. AGO (Arbeitsgemeinschaft Gynäkologische Onkologie, Organgruppe Mamma); 2009: http://www.ago-online.org

\section{KEY MESSAGES}

- Locoregional recurrence of breast cancer should be treated curatively.

- With metastatic breast cancer, restoration of quality of life, reduction of tumor-related symptoms, and maintenance of the patient's social environment are the priorities; prolonging life is a secondary aim.

- Multimodal therapies increase remission rates and progression-free intervals.

- Treatment should continue for as long as the benefits are greater than the unwanted side effects.

- All patients should be offered the opportunity to take part in clinical studies. 
7. NCCN (National Comprehensive Cancer Network): NCCN Practice Guidelines in Oncology: Breast Cancer 2009: http://www.nccn.org

8. Cochrane Collaboration 2009: http://www.cochrane.org

9. Wapnir IL, Anderson SJ, Mamounas EP, et al.: Prognosis after ipsilateral breast tumor recurrence and locoregional recurrences in five National Surgical Adjuvant Breast and Bowel Project node-positive adjuvant breast cancer trials. J Clin Oncol 2006; 24: 2028-37.

10. Waeber M, Castiglione-Gertsch M, Dietrich D, et al.: Adjuvant therapy after excision and radiation of isolated postmastectomy locoregional breast cancer recurrence: definitive results of a phase III randomized trial (SAKK 23/82) comparing tamoxifen with observation. Ann Oncol 2003; 14: 1215-21.

11. Mauri D, Polyzos NP, Salanti G, Pavlidis N, loannidis JP: Multiple-treatments meta-analysis of chemotherapy and targeted therapies in advanced breast cancer. J Natl Cancer Inst 2008; 17: 1780-91.

12. Gennari A, Conte P, Rosso R, Orlandini C, Bruzzi P: Survival of metastatic breast carcinoma patients over a 20 -year period: a retrospective analysis based on individual patient data from six consecutive studies. Cancer 2005; 104: 1742-50.

13. Ruiterkamp J, Ernst MF, van de Poll-Franse LV, et al.: Surgical resection of the primary tumour is associated with improved survival in patients with distant metastatic breast cancer at diagnosis. Eur J Surg Oncol 2009; 35: 1146-51.

14. Sledge GWJ, Hu P, Falkson G, Tormey D, Abeloff M: Comparison of chemotherapy with chemohormonal therapy as first-line therapy for metastatic, hormone-sensitive breast cancer: An Eastern Cooperative Oncology Group study. J Clin Oncol 2000; 18: 262-6.

15. Rasmussen BB, Regan MM, Lykkesfeldt AE, et al.: Adjuvant letrozole versus tamoxifen according to centrally-assessed ERBB2 status for postmenopausal women with endocrine-responsive early breast cancer: supplementary results from the BIG 1-98 randomised trial. Lancet Oncol 2008; 9: 23-8.

16. Kaufman B, Mackey JR, Clemens MR, et al.: Trastuzumab plus anastrozole versus anastrozole alone for the treatment of postmenopausal women with human epidermal growth factor receptor 2-positive, hormone receptor-positive metastatic breast cancer. Results from the randomized phase III TAnDEM study. J Clin Oncol 2009; 27: 5529-37.
17. Johnston S, Pippen J Jr, Pivot X, et al.: Lapatinib combined with letrozole versus letrozole and placebo as first-line therapy for postmenopausal hormone receptor-positive metastatic breast cancer. J Clin Oncol 2009; 27: 5538-46.

18. Sledge GW, Neuberg D, Bernardo P, et al.: Phase III trial of doxorubicin, paclitaxel, and the combination of doxorubicin and paclitaxel as frontline chemotherapy for metastatic breast cancer: an intergroup trial (E1193). J Clin Oncol 2003; 21: 588-92

19. Jones SE, Erban J, Overmoyer B, et al.: Randomized phase III study of docetaxel compared with paclitaxel in metastatic breast cancer. J Clin Oncol 2005; 23: 5542-51.

20. Miller K, Wang M, Gralow J, et al.: Paclitaxel plus bevacizumab versus paclitaxel alone for metastatic breast cancer. N Engl J Med 2007; 357: 2666-76

21. Slamon DJ, Leyland-Jones B, Shak S, et al.: Use of chemotherapy plus a monoclonal antibody against HER2 for metastatic breast cancer that overexpresses HER2. N Engl J Med 2001; 344: 783-92.

22. Di Leo A, Gomez HL, Aziz Z, et al.: Phase III, double-blind, randomized study comparing lapatinib plus paclitaxel with placebo plus paclitaxel as first-line treatment for metastatic breast cancer. J Clin Oncol 2008; 26: 5544-52.

23. Hillner BE, Ingle JN, Chlebowski RT, et al.: American Society of Clinical Oncology 2003 update on the role of bisphosphonates and bone health issues in women with breast cancer. J Clin Oncol 2003; 21 : 4042-57.

24. Gnant M, Mlineritsch B, Schippinger W, et al.: Endocrine therapy plus zoledronic acid in premenopausal breast cancer. N Engl J Med 2009; 360: 679-91.

25. Tan C, Sedrakyan A, Browne J, Swift S, Treasure T: The evidence on the effectiveness of management for malignant pleural effusion: a systematic review. Eur J Cardiothorac Surg 2006; 29: 829-38.

\section{Corresponding author}

Prof. Dr. med. Bernd Gerber

Universitäts-Frauenklinik Rostock

Südring 81

18059 Rostock, Germany

bernd.gerber@med.uni-rostock.de

For e-references please refer to: www.aerzteblatt-international.de/ref0610 


\title{
Recurrent Breast Cancer
}

\author{
Treatment Strategies for Maintaining and Prolonging a Good Quality of Life
}

\author{
Bernd Gerber, Mathias Freund, Toralf Reimer
}

\section{E-References}

e1. Robert Koch Institut: Evaluation of cancer incidence in germany (2003-2004); 2009: http://www.rki.de/GBE/KREBS/KREBS.HTM

e2. SEER, 2009: http://seer.cancer.gov/

e3. Saphner T, Tormey DC, Gray R: Annual hazard rates of recurrence for breast cancer after primary therapy. J Clin Oncol 1996; 14: 2738-46.

e4. Christiansen P, Al Suliman N, Bjerre K, Moller S: Recurrence pattern and prognosis in low-risk breast cancer patients-data from the DBCG 89-A programme. Acta Oncol 2008; 47: 691-703.

e5. Lin NU, Claus E, Sohl J, et al.: Sites of distant recurrence and clinical outcomes in patients with metastatic triple-negative breast cancer: high incidence of central nervous system metastases. Cancer 2008; 113: 2638-45.

e6. Kaplan HG, Malmgren JA: Impact of triple negative phenotype on breast cancer prognosis. Breast J 2008; 14: 456-63.

e7. Oxford Centre for Evidence-based Medicine Levels of Evidence: Levels of evidence and grades of recommendation. http://www cebm net/levels_of_evidence asp, 2006.

e8. Broom RJ, Tang PA, Simmons C, et al.: Changes in estrogen receptor, progesterone receptor and her-2/neu status with time: discordance rates between primary and metastatic breast cancer. Anticancer Res 2009; 29: 1557-62.

e9. Wurschmidt F, Dahle J, Petersen C, et al.: Reirradiation of recurrent breast cancer with and without concurrent chemotherapy. Radiat Oncol 2008; 3: 28.

e10. Wapnir IL, Aebi S, Geyer CE, et al.: A randomized clinical trial of adjuvant chemotherapy for radically resected locoregional relapse of breast cancer: IBCSG 27-02, BIG 1-02, and NSABP B-37. Clin Breast Cancer 2008; 8: 287-92.

e11. Rauschecker H, Clarke M, Gatzemeier W, Recht A: Systemic therapy for treating locoregional recurrence in women with breast cancer. Cochrane Database Syst Rev 2001; CD002195.

e12. Semrau S, Gerber B, Reimer T, Klautke G, Fietkau R: Concurrent radiotherapy and taxane chemotherapy in patients with locoregional recurrence of breast cancer. A retrospective analysis. Strahlenther Onkol 2006; 182: 596-603.

e13. Pergolizzi S, Adamo V, Russi E, et al.: Prospective multicenter study of combined treatment with chemotherapy and radiotherapy in breast cancer women with the rare clinical scenario of ipsilateral supraclavicular node recurrence without distant metastases. Int J Radiat Oncol Biol Phys 2006; 65: 25-32.

e14. Schlesinger-Raab A C, Eckel R, Engel J, Sauer H, Löhrs U, Molls M, Hölzel D: Metastasiertes Mammakarzinom: Keine Lebensverlängerung seit 20 Jahren. Dtsch Arztebl 2005; 102(40): A 2706-14.

e15. Wilcken N, Dear R: Chemotherapy in metastatic breast cancer: A summary of all randomized trials reported 2000-2007. Eur J Cancer 2008; 44: 2218-25.

e16. Giordano SH, Buzdar AU, Smith TL, et al.: Is breast cancer survival improving? Cancer 2004; 100: 44-52.

e17. Andre F, Slimane K, Bachelot T, et al: Breast cancer with synchronous metastases: trends in survival during a 14 -year period. J Clin Oncol 2004; 22: 3302-8. e18. Chia SK, Speers CH, D'yachkova Y, et al.: The impact of new chemotherapeutic and hormone agents on survival in a populationbased cohort of women with metastatic brest cancer. Cancer 2007; 110: 973-9.

e19. Therasse P, Arbuck SG, Eisenhauer EA, et al:: New guidelines to evaluate the response to treatment in solid tumors. European Organization for Research and Treatment of Cancer, National Cancer Institute of the United States, National Cancer Institute of Canada. J Natl Cancer Inst 2000; 92: 205-16.

e20. Telli ML, Hunt SA, Carlson RW, Guardino AE: Trastuzumab-related cardiotoxicity: calling into question the concept of reversibility. J Clin Oncol 2007; 25: 3525-33.

e21. Rapiti E, Verkooijen HM, Vlastos G, et al.: Complete excision of primary breast tumor improves survival of patients with metastatic breast cancer at diagnosis. J Clin Oncol 2006; 24: 2743-9.

e22. Babiera GV, Rao R, Feng L, et al.: Effect of primary tumor extirpation in breast cancer patients who present with stage IV disease and an intact primary tumor. Ann Surg Oncol 2006; 13: 776-82.

e23. Montemurro F, Rondon G, Ueno NT, et al.: Factors affecting progression-free survival in hormone-dependent metastatic breast cancer patients receiving high-dose chemotherapy and hematopoietic progenitor cell transplantation: role of maintenance endocrine therapy. Bone Marrow Transplant 2002; 29: 861-6.

e24. Carlson RW: Sequencing of endocrine therapies in breast cancer - integration of recent data. Breast Cancer Res Treat 2002; 75 Suppl 1: S27-S32.

e25. Klijn JG, Blamey RW, Boccardo F, et al.: Combined tamoxifen and luteinizing hormone-releasing hormone (LHRH) agonist versus LHRH agonist alone in premenopausal advanced breast cancer: a meta-analysis of four randomized trials. J Clin Oncol 2001; 19: 343-53.

e26. Forward DP, Cheung KL, Jackson L, Robertson JF: Clinical and endocrine data for goserelin plus anastrozole as second-line endocrine therapy for premenopausal advanced breast cancer. $\mathrm{Br}$ J Cancer 2004; 90: 590-4.

e27. Fossati R, Confalonieri C, Torri V, et al.: Cytotoxic and hormonal treatment for metastatic breast cancer: a systematic review of published randomized trials involving 31,510 women. J Clin Oncol 1998; 16: 3439-60.

e28. Albain KS, Nag SM, Calderillo-Ruiz G, et al.: Gemcitabine plus paclitaxel versus paclitaxel monotherapy in patients with metastatic breast cancer and prior anthracycline treatment. J Clin Oncol 2008; 26: 3950-7.

e29. O'Shaughnessy J, Miles D, Vukelja S, et al.: Superior survival with capecitabine plus docetaxel combination therapy in anthracycline-pretreated patients with advanced breast cancer: phase III trial results. J Clin Oncol 2002; 20: 2812-23.

e30. Jones D, Ghersi D, Wilcken N: Addition of drug/s to a chemotherapy regimen for metastatic breast cancer. Cochrane Database Syst Rev 2006; 3: CD003368.

e31. Sparano JA, Wang M, Martino S, et al.: Weekly paclitaxel in the adjuvant treatment of breast cancer. N Engl J Med 2008; 358: 1663-71.

e32. Seidman AD, Berry D, Cirrincione C, et al.: Randomized phase III trial of weekly compared with every-3-weeks paclitaxel for metastatic breast cancer, with trastuzumab for all HER-2 overexpres- 
sors and random assignment to trastuzumab or not in HER-2 nonoverexpressors: final results of Cancer and Leukemia Group B protocol 9840. J Clin Oncol 2008; 26: 1642-9.

e33. Gradishar WJ, Tjulandin S, Davidson N, et al.: Phase III trial of nanoparticle albumin-bound paclitaxel compared with polyethylated castor oil-based paclitaxel in women with breast cancer. J Clin Oncol 2005; 23: 7794-803.

e34. Perez EA: Novel enhanced delivery taxanes: an update. Semin Oncol 2007; 34: suppl-5.

e35. Robinson DM, Keating GM: Albumin-bound Paclitaxel: in metastatic breast cancer. Drugs 2006; 66: 941-8.

e36. Bhushan S, Walko CM: Ixabepilone: a new antimitotic for the treatment of metastatic breast cancer. Ann Pharmacother 2008; 42: 1252-61

e37. Lechleider RJ, Kaminskas E, Jiang X, et al.: Ixabepilone in combination with capecitabine and as monotherapy for treatment of advanced breast cancer refractory to previous chemotherapies. Clin Cancer Res 2008; 14: 4378-84.

e38. Bunnell C, Vahdat L, Schwartzberg $L$, et al.: Phase I/II study of ixabepilone plus capecitabine in anthracycline-pretreated/resistant and taxane-resistant metastatic breast cancer. Clin Breast Cancer 2008; 8: 234-41.

e39. Schneider BP, Wang M, Radovich M, et al.: Association of vascular endothelial growth factor and vascular endothelial growth factor receptor-2 genetic polymorphisms with outcome in a trial of paclitaxel compared with paclitaxel plus bevacizumab in advanced breast cancer: ECOG 2100. J Clin Oncol 2008; 26: 4672-8.

e40. Marty M, Cognetti F; D Maraninchi D, et al.: Randomized phase II trial of the efficacy and safety of trastuzumab combined with docetaxel in patients with human epidermal growth factor receptor 2-positive metastatic breast cancer administered as first-line treatment: the M77001 study group. J Clin Oncol 2005; 23: 4247-50.

e41. Geyer CE, Forster J, Lindquist D, et al.: Lapatinib plus capecitabine for HER2-positive advanced breast cancer. N Engl J Med 2006; 355: 2733-43.

e42. Press MF, Finn RS, Cameron D, et al.: HER-2 Gene amplification, HER-2 and epidermal growth factor receptor mRNA and protein expression, and lapatinib efficacy in women with metastatic breast cancer. Clin Cancer Res 2008; 14: 7861-70.

e43. Cameron D, Casey M, Press M, et al.: A phase III randomized comparison of lapatinib plus capecitabine versus capecitabine alone in women with advanced breast cancer that has progressed on trastuzumab: updated efficacy and biomarker analyses. Breast Cancer Res Treat 2008; 112: 533-43.

e44. von Minckwitz G, du BA, Schmidt M, et al.: Trastuzumab beyond progression in human epidermal growth factor receptor 2-positive advanced breast cancer: a german breast group 26/breast international group 03-05 study. J Clin Oncol 2009; 27: 1999-2006.

e45. Portera CC, Walshe JM, Rosing DR, et al.: Cardiac toxicity and efficacy of trastuzumab combined with pertuzumab in patients with [corrected] human epidermal growth factor receptor 2-positive metastatic breast cancer. Clin Cancer Res 2008; 14: 2710-6.

e46. Diel IJ, Body JJ, Lichinitser MR, et al.: Improved quality of life after long-term treatment with the bisphosphonate ibandronate in patients with metastatic bone disease due to breast cancer. Eur J Cancer 2004; 40: 1704-12.

e47. Diel IJ: Effectiveness of bisphosphonates on bone pain and quality of life in breast cancer patients with metastatic bone disease: a review. Support Care Cancer 2007; 15: 1243-9.

e48. Ha TC, Li H: Meta-analysis of clodronate and breast cancer survival. Br J Cancer 2007; 96: 1796-801.

e49. Aapro M, Abrahamsson PA, Body JJ, et al.: Guidance on the use of bisphosphonates in solid tumours: recommendations of an international expert panel. Ann Oncol 2008; 19: 420-32.

e50. Pavlakis N, Schmidt R, Stockler M: Bisphosphonates for breast cancer. Cochrane Database Syst Rev 2005; CD003474.

e51. Ross JR, Saunders Y, Edmonds PM, et al.: Systematic review of role of bisphosphonates on skeletal morbidity in metastatic cancer. BMJ 2003; 327: 469 e52. Body JJ, Diel IJ, Bell R, et al.: Oral ibandronate improves bone pain and preserves quality of life in patients with skeletal metastases due to breast cancer. Pain 2004; 111: 306-12.

e53. Lipton A, Steger GG, Figueroa J, et al.: Randomized activecontrolled phase II study of denosumab efficacy and safety in patients with breast cancer-related bone metastases. J Clin Onco 2007; 25: 4431-7.

e54. Patchell RA, Tibbs PA, Regine WF, et al.: Direct decompressive surgical resection in the treatment of spinal cord compression caused by metastatic cancer: a randomized trial. Lancet 2005; 366: 643-8.

e55. Ibrahim A, Crockard A, Antonietti P, et al.: Does spinal surgery improve the quality of life for those with extradural (spinal) osseous metastases? An international multicenter prospective observational study of 223 patients. Invited submission from the Joint Section Meeting on Disorders of the Spine and Peripheral Nerves, March 2007. J Neurosurg Spine 2008; 8: 271-8.

e56. Chinot OL: Cerebral metastases. Rev Prat 2006; 56: 1799-804.

e57. Rath GK, Julka PK, Thulkar S, et al.: Radiofrequency ablation of hepatic metastasis: results of treatment in forty patients. J Cancer Res Ther 2008; 4: 14-7.

e58. Vogl TJ, Naguib NN, Eichler K, et al.: Volumetric evaluation of liver metastases after thermal ablation: long-term results following MR-guided laser-induced thermotherapy. Radiology 2008; 249 : 865-71.

e59. Kuvshinoff B, Fong Y: Surgical therapy of liver metastases. Semin Oncol 2007; 34: 177-85

e60. Demicheli R, Retsky MW, Hrushesky WJ, Baum M, Gukas ID: The effects of surgery on tumor growth: a century of investigations. Ann Oncol 2008; 19: 1821-8.

e61. Shaw P, Agarwal R: Pleurodesis for malignant pleural effusions. Cochrane Database Syst Rev 2004; CD002916.

e62. Gibson LJ, Dawson CK, Lawrence DH, Bliss JM: Aromatase inhibitors for treatment of advanced breast cancer in postmenopausal women. Cochrane Database Syst Rev 2007; CD003370.

e63. Ingle JN: Sequencing of endocrine therapy in postmenopausal women with advanced breast cancer. Clin Cancer Res 2004: 10: 362S-7S.

e64. Iaffaioli RV, Formato R, Tortoriello A, et al.: Phase II study of sequential hormonal therapy with anastrozole/exemestane in ad-vanced and metastatic breast cancer. Br J Cancer 2005; 92: 1621-5.

e65. Carlini P, Michelotti A, Ferretti G, et al.: Clinical evaluation of the use of exemestane as further hormonal therapy after nonsteroidal aromatase inhibitors in postmenopausal metastatic breast cancer patients. Cancer Invest 2007; 25: 102-5

e66. Gennatas C, Michalaki V, Carvounis E, et al.: Third-line hormonal treatment with exemestane in postmenopausal patients with advanced breast cancer progressing on letrozole or anastrozole. A phase II trial conducted by the Hellenic Group of Oncology (HELGO). Tumori 2006; 92: 13-7.

e67. Vergote I, Amant F, Leunen K, et al.: Metastatic breast cancer: sequencing hormonal therapy and positioning of fulvestrant. Int J Gynecol Cancer 2006; 16 Suppl 2: 524-6.

e68. Bartsch R, Mlineritsch B, Gnant M, et al.: The Austrian fulvestrant registry: results from a prospective observation of fulvestrant in postmenopausal patients with metastatic breast cancer. Breast Cancer Res Treat 2009; 115: 373-80.

e69. Chia S, Gradishar W, Mauriac L, et al.: Double-blind, randomized placebo controlled trial of fulvestrant compared with exemestane after prior nonsteroidal aromatase inhibitor therapy in postmenopausal women with hormone receptor-positive, advanced breast cancer: results from EFECT. J Clin Oncol 2008; 26: 1664-70.

e70. Vergote I, Abram P: Fulvestrant, a new treatment option for advanced breast cancer: telerability versus existing agents. Ann Oncol 2006; 17: 200-4. 